\title{
Search for Excited Leptons at HERA
}

\author{
TRINH Thi Nguyet \\ On behalf of the H1 Collaboration
}

Centre de Physique des Particules de Marseille

163 Avenue de Luminy, F-13288 Marseille Cedex 9, France

\begin{abstract}
Searches for excited electrons and neutrinos have been performed using the complete HERA I and II data samples collected by the H1 detector at $\sqrt{s}=320 \mathrm{GeV}$ corresponding to an integrated luminosity of up to $435 \mathrm{pb}^{-1}$. In absence of a signal, the limits on the ratio of the coupling to the compositeness scale derived extend the excluded region to higher masses than has been possible in previous searches.
\end{abstract}

\section{Introduction}

Compositeness models [2] attempt to explain the hierarchy of masses in the Standard Model (SM) by the existence of a substructure within the fermions. Several of these models predict excited states of the known leptons. Excited leptons $\left(F^{*}\right)$ are assumed to have the same electroweak SU(2) and U(1) gauge couplings, $g$ and $g^{\prime}$, to the vector bosons, but are expected to be grouped into both left- and right-handed weak isodoublets with vector couplings. The existence of the right-handed doublets is required to protect the ordinary light leptons from radiatively acquiring a large anomalous magnetic moment via the $F^{*} F V$ interaction (where $\mathrm{V}$ is a $\gamma, Z$, or $W)$. Considering only the electroweak interaction, the phenomenological model describes this interaction by the Lagrangian density:

$$
L_{F^{*} F}=\frac{1}{2 \Lambda} \bar{F}_{R}^{*} \sigma^{\mu \nu}\left[g f \frac{\vec{\tau}}{2} \partial_{\mu} \vec{W}_{\nu}+g^{\prime} f^{\prime} \frac{Y}{2} \partial_{\mu} B_{\nu}\right] F_{L}+h . c .
$$

where the new weights $f$ and $f^{\prime}$ multiply the standard coupling constants $g$ and $g^{\prime}$ corresponding to the weak $\mathrm{SU}(2)$ and the electromagnetic $\mathrm{U}(1)$ sectors respectively. The matrix $\sigma^{\mu \nu}$ is the covariant bilinear tensor, $\tau$ are the Pauli matrices, $W_{\mu \nu}$ and $B_{\mu \nu}$ represent the fully gauge invariant field tensors, and $\mathrm{Y}$ is the weak hypercharge. The parameter $\Lambda$ has units of energy and can be regarded as the compositeness scale. The relative values of $f$ and $f^{\prime}$ affect the size of the single-production cross section, their detection efficiencies and also the branching ratios of excited leptons.

Excited electrons and neutrinos may be produced in electron(positron)-proton collisions at HERA via $t$-channel $\gamma(Z)$ or $W^{ \pm}$gauge boson exchange. In the case of excited neutrinos, the cross section is much larger in $e^{-} p$ collsions than in $e^{+} p$ collsions due to the favourable valence u-quark and the helicity enhancement, specific to CC-like processes. Therefore the search for excited neutrinos uses only $e^{-} p$ sample data with an integrated luminosity of $184 \mathrm{pb}^{-1}$. In the case of excited electrons, both $e^{-} p$ and $e^{+} p$ collision modes are used, corresponding to total integrated luminosity of $435 \mathrm{pb}^{-1}$.

\section{Data analysis and results}

Excited leptons $(l=e, \nu)$ are searched for in the following decay channels: $l^{*} \rightarrow l \gamma, l^{*} \rightarrow l Z$, $l^{*} \rightarrow l W$. The final states resulting from the $Z$ or $W$ hadronic decays are taken into account 
Search for $\mathrm{e}^{*}, \nu^{*}$ HERA I+II $(\sqrt{s}=320 \mathrm{GeV}$, preliminary $)$

\begin{tabular}{|c||c|c||c|}
\hline Selection & Data & SM & Efficiency $\times$ BR \\
\hline$e^{*} \rightarrow \nu W_{\hookrightarrow q q}$ & 172 & $175 \pm 39$ & $\sim 40 \%$ \\
\hline$e^{*} \rightarrow e Z_{\hookrightarrow q q}$ & 351 & $318 \pm 64$ & $\sim 45 \%$ \\
\hline$e^{*} \rightarrow e \gamma$ & 112 & $125 \pm 19$ & $60-70 \%$ \\
\hline \hline$\nu^{*} \rightarrow \nu \gamma$ & 9 & $15 \pm 4$ & $50 \%$ \\
\hline$\nu^{*} \rightarrow e W_{\hookrightarrow q q}$ & 198 & $189 \pm 33$ & $30-40 \%$ \\
\hline$\nu^{*} \rightarrow \nu Z_{\hookrightarrow q q}$ & 111 & $102 \pm 24$ & $40 \%$ \\
\hline$\nu^{*} \rightarrow e W_{\hookrightarrow \nu \mu}$ & 0 & $0.54 \pm 0.04$ & $3-4.5 \%$ \\
\hline$\nu^{*} \rightarrow e W_{\hookrightarrow \nu e}$ & 0 & $0.6 \pm 0.3$ & $4-6 \%$ \\
\hline$\nu^{*} \rightarrow \nu Z_{\hookrightarrow e e}$ & 0 & $0.12 \pm 0.04$ & $2 \%$ \\
\hline
\end{tabular}

Table 1: Observed and predicted event yields for the event classes of $e^{*}$ and $\nu^{*}$ searches. The selection efficiency for the signal multiplied by the branching ratio (BR) in each decay channel is also presented.

for both excited electrons and neutrinos and the $Z$ or $W$ leptonic decays are taken into account only for excited neutrinos. In the following, the selection criteria are described for the decay channels.

\section{The $\nu^{*} \rightarrow \nu \gamma$ channel}

Candidate events are selected by requiring missing transverse momentum $P_{T}^{\text {miss }}>15 \mathrm{GeV}$, where the photon is identified as an isolated electromagnetic (e.m.) cluster in the LAr calorimeter within a polar angle of $5^{\circ}$ to $120^{\circ}$. The photon candidates measured within the acceptance of the central tracker $\left(\theta^{\gamma}>20^{\circ}\right)$ are required to have no associated tracks. The neutral current (NC) and charged current (CC) backgrounds are reduced by imposing the longitudinal momentum balance $E-P_{Z}>45 \mathrm{GeV}$ for events with photon candidates at lower transverse momentum $P_{T}^{\gamma}<40 \mathrm{GeV}$ and by requiring the virtuality $\left(Q_{\gamma}^{2}\right)$ to satisfy $\log \left(Q_{\gamma}^{2}\right)>3.5 \mathrm{GeV}^{2}$. The background is further suppressed by rejecting events with a transverse momentum of the final hadronic in the calorimeter $P_{T}^{h}<5 \mathrm{GeV}$.

\section{The $e^{*} \rightarrow e \gamma$ channel}

Candidate events are selected with two isolated e.m. clusters in the LAr calorimeter of transverse energy greater than $20 \mathrm{GeV}$ and $15 \mathrm{GeV}$, respectively, and with a polar angle between $5^{\circ}$ and $130^{\circ}$. The sum of the energies of the two clusters has to be greater than $100 \mathrm{GeV}$. The background from NC is further suppressed by rejecting events with a total transverse energy of the two isolated e.m. clusters lower than $75 \mathrm{GeV}$.

\section{The $e^{*} \rightarrow e Z, \nu W$ and $\nu^{*} \rightarrow e W, \nu Z$ channels with $Z, W \rightarrow q q^{\prime}$}

These channels use subsample of events with at least two jets with high transverse momentum $P_{T}^{j 1(j 2)}>20(15) \mathrm{GeV}$ reconstructed within $5^{\circ}<\theta^{j 1(j 2)}<130^{\circ}$. The dijet invariant mass must be compatible with the relevant boson mass and should be closest to them.

Events with two high $P_{T}$ jets and one electron: The channels $e^{*} \rightarrow e Z_{\rightarrow q q}$ and $\nu^{*} \rightarrow$ $e W_{\rightarrow q q}$ are characterised by at least two high $P_{T}$ jets and an energetic isolated e.m. cluster $P_{T}^{e}>10 \mathrm{GeV}\left(P_{T}^{e}>20 \mathrm{GeV}\right.$ for $\left.e^{*} \rightarrow e Z_{\rightarrow q q}\right)$ in the polar angle $5^{\circ}<\theta^{e}<130^{\circ}$. The 
polar angle of the highest $P_{T}$ jet resulting from $W$ boson should be lower than $80^{\circ}$. The dijet invariant mass has to be greater than $60 \mathrm{GeV}$. If $P_{T}^{e}<65 \mathrm{GeV}$, the dijet invariant mass must be greater than $75 \mathrm{GeV}$. In the case of the $\nu^{*} \rightarrow e W_{\rightarrow q q}$ channel, to reduce the $\mathrm{NC}$ background, the polar angle of e.m. cluster must be lower than $90^{\circ}$. Furthermore, the background is reduced by requiring the virtuality computed from the e.m. cluster kinematics $Q^{2}>2500 \mathrm{GeV}^{2}$ if $P_{T}^{e}<25 \mathrm{GeV}$ and by requiring a third jet with $P_{T}>5 \mathrm{GeV}$ to be present in the event if $P_{T}^{e}>50 \mathrm{GeV}$.
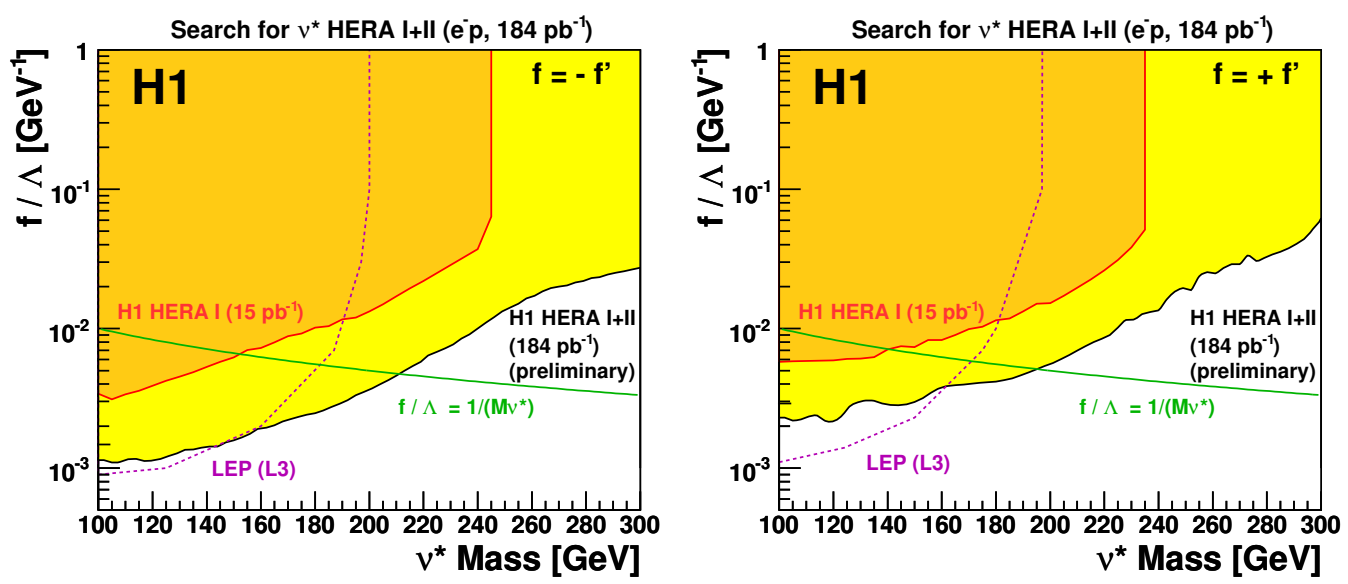

Figure 1: The 95\% C.L. limits obtained for coupling constants $(f / \Lambda)$ as a function of the excited neutrino mass within two assumptions: $f=-f^{\prime}$ (left) and $f=+f^{\prime}$ (right). The observed limits from this annalysis using all $\mathrm{H} 1 e^{-} p$ data is presented by the yellow area. Values of the couplings above the curves are excluded. The orange-dark area corresponds to the exclusion domain published by the H1 experiment using 98/99 data and the dashed line to the exclusion limit from the L3 experiment at LEP [4].

Events with two high $P_{T}$ jets and $P_{T}^{\text {miss }}$ : The channels $e^{*} \rightarrow \nu W_{\rightarrow q q}$ and $\nu^{*} \rightarrow \nu Z_{\rightarrow q q}$ are characterised by at least two high jets and $P_{T}^{\text {miss }}>12 \mathrm{GeV}$. In the case of $e^{*} \rightarrow \nu W \rightarrow q q$ channel, the ratio $V_{a p} / V_{p}$ of transverse energy flow anti-parallel and parallel to the hadronic final state [3] is required to be lower than 0.3 to reject the photoproduction $(\gamma p)$ background. The dijet invariant mass is required to be greater than $50 \mathrm{GeV}$. Furthermore, if $P_{T}^{\text {miss }}<65 \mathrm{GeV}$ the dijet is required to have an invariant mass above $65 \mathrm{GeV}$. In the case of the $\nu^{*} \rightarrow \nu Z_{\rightarrow q q}$ channel, a dijet invariant mass greater than $60 \mathrm{GeV}$ is required. In order to reduce $\mathrm{CC}$ background, the total hadronic system is required to have a polar angle above $20^{\circ}$ and a third jet with $P_{T}>5 \mathrm{GeV}$ has to be present in the event if $P_{T}^{\text {miss }}<65 \mathrm{GeV}$. The longitudinal balance $E-P_{Z}>25 \mathrm{GeV}$ is imposed if $P_{T}^{m i s s}<50 \mathrm{GeV}$. In addition, events with $P_{T}^{m i s s}<30 \mathrm{GeV}$ are only accepted if the topological variable $V_{a p} / V_{p}>0.1$.

The $\nu^{*} \rightarrow e W, \nu Z$ channels with $Z \rightarrow e e, W \rightarrow \nu e(\mu)$

Events with two electron and $P_{T}^{\text {miss }}$ : These channels use a subsample of events with two high $P_{T}$ isolated e.m. clusters $P_{T}^{e 1(e 2)}>20(15) \mathrm{GeV}$ and a polar angle $5^{\circ}<\theta^{e 1(e 1)}<100^{\circ}\left(120^{\circ}\right)$ and $P_{T}^{\text {miss }}>12 \mathrm{GeV}$. The clusters are required to have associated tracks if they are measured within the acceptance of the central tracker. 
Events with one muon and an electron: Candidate events containing an islolated muon plus an isolated electron, both having a high transverse momentum $P_{T}^{e(\mu)}>20(10) \mathrm{GeV}$ and a polar angle $5^{\circ}<\theta^{e(\mu)}<100^{\circ}\left(160^{\circ}\right)$ are selected. A cut $P_{T}^{\text {miss }}>12 \mathrm{GeV}$ is applied. The backgrounds are reduced by requiring the virtuality $\left(Q_{e}^{2}\right)$ to satisfy $\log \left(Q_{e}^{2}\right)>3 \mathrm{GeV}^{2}$.

The observed number of events are compared to the expected SM background in table 1 for each search channel. A good overall agreement is found for all channels. No significant deviation is observed in the data. The selection efficiency for each decay channel for the both $e^{*}$ and $\nu^{*}$ search is presented also in the table 1 .

\section{Interpretation and Conclusions}

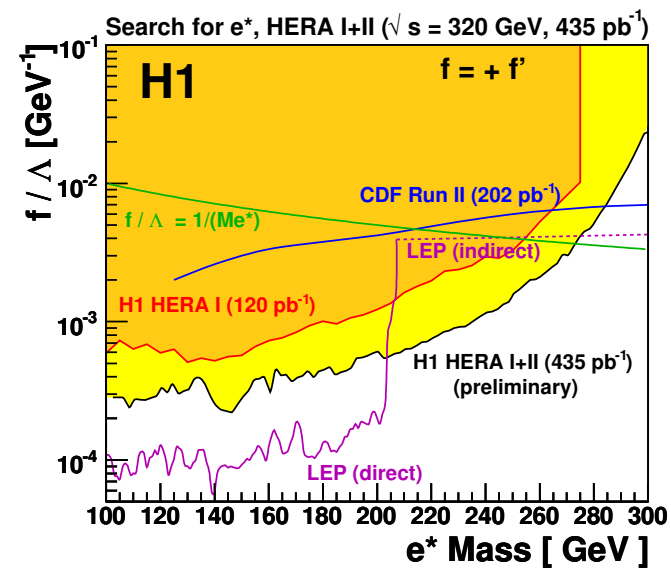

Figure 2: The $95 \%$ C.L. limits obtained for coupling constants $(f / \Lambda)$ as a function of the excited electron mass within assumption: $f=+f^{\prime}$. The observed limit from this analysis using $435 p b^{-1}$ of $\mathrm{H} 1$ data is presented by the yellow area. The orangedark area corresponds to the exclusion domain published by the $\mathrm{H} 1$ experiment using HERA I data. The combined exclusion limit from LEP experiments is presented by the violet line. The result of the CDF [5] experiment at the Tevatron is also shown.

In absence of a signal for both excited electron and neutrino production, upper limits on the coupling $f / \Lambda$ have been derived at $95 \%$ Confidence Level (C.L.) as a function of excited electron and neutrino masses. In case of excited neutrinos production, the obtained limits are displayed for the two assumptions $f=-f^{\prime}$ and $f=+f^{\prime}$ (figure 1). For $f=-f^{\prime}$ (maximal $\gamma \nu * \nu$ coupling) and assuming $f / \Lambda=1 / M_{\nu *}$, excited neutrinos with masses below $211 \mathrm{GeV}$ are excluded at $95 \%$ C.L. The limits on the ratio $f / \Lambda$ also are given for the excited electron for the hypothesis $f=+f^{\prime}$ (figure 2). We do not consider the case $f=-f^{\prime}$, because the $\gamma e^{*} e$ coupling constant would be equal to zero and the production cross section of the excited electron is very small. For this hypothesis and assuming $f / \Lambda=1 / M_{e^{*}}$, excited electrons with masses below $273 \mathrm{GeV}$ are excluded at 95\% C.L.

\section{References}

[1] Slides http://indico. cern. ch/contributionDisplay . py? contribId=131\&sessionId=3\&conf Id=9499

[2] U. Baur, M. Spira and P. M. Zerwas, Phys. Rev. D 42 (1990) 815.

[3] C. Adloff et al. [H1 Collaboration], Phys. Lett. B 525 (2002) 9, [hep-ex/0110037].

[4] M. Acciarri et al. [L3 Collaboration], Phys. Lett. B 502 (2001) 37, [hep-ex/0011068].

[5] D. Acosta et al. [CDF Collaboration], Phys. Rev. Lett. 94 (2005) 101802, [hep-ex/0410013]. 